\title{
What Players Want: Information Needs of Players on Post-Game Visualizations
}

\author{
Günter Wallner \\ Department of Industrial Design \\ Eindhoven University of Technology \\ Eindhoven, The Netherlands \\ Johannes Kepler University Linz \\ Linz, Austria \\ g.wallner@tue.nl \\ Regina Bernhaupt \\ Department of Industrial Design \\ Eindhoven University of Technology \\ Eindhoven, The Netherlands \\ r.bernhaupt@tue.nl
}

\author{
Marnix van Wijland \\ Department of Industrial Design \\ Eindhoven University of Technology \\ Eindhoven, The Netherlands \\ m.j.v.wijland@student.tue.nl
}

\author{
Simone Kriglstein \\ Masaryk University \\ Brno, Czech Republic \\ AIT Austrian Institute of Technology GmbH \\ Vienna, Austria \\ Faculty of Computer Science \\ University of Vienna \\ Vienna, Austria \\ kriglstein@mail.muni.cz
}

\begin{abstract}
With the rise of competitive online gaming and esports, players' ability to review, reflect upon, and improve their in-game performance has become important. Post-play visualizations are key for such improvements. Despite the increased interest in visualizations of gameplay, research specifically informing the design of playercentric visualizations is currently limited. As with all visualizations, their design should, however, be guided by a thorough understanding of the goals to be achieved and which information is important and why.

This paper reports on a mixed-methods study exploring the information demands posed by players on post-play visualizations and the goals they pursue with such visualizations. We focused on three genres that enjoy great popularity within the competitive gaming scene. Our results provide useful guideposts on which data to focus on by offering an overview of the relevance of different in-game metrics across genres. Lastly, we outline high-level implications for the design of post-play visualizations.
\end{abstract}

\section{CCS CONCEPTS}

- Applied computing $\rightarrow$ Computer games; $\bullet$ Human-centered computing $\rightarrow$ Information visualization; Empirical studies in visualization.

Permission to make digital or hard copies of all or part of this work for personal or classroom use is granted without fee provided that copies are not made or distributed for profit or commercial advantage and that copies bear this notice and the full citation on the first page. Copyrights for components of this work owned by others than the author(s) must be honored. Abstracting with credit is permitted. To copy otherwise, or republish, to post on servers or to redistribute to lists, requires prior specific permission and/or a fee. Request permissions from permissions@acm.org.

CHI '21, May 8-13, 2021, Yokohama, Japan

(c) 2021 Copyright held by the owner/author(s). Publication rights licensed to ACM. ACM ISBN 978-1-4503-8096-6/21/05 .. \$15.00

https://doi.org/10.1145/3411764.3445174

\section{KEYWORDS}

games, players, gameplay visualization, information needs

\section{ACM Reference Format:}

Günter Wallner, Marnix van Wijland, Regina Bernhaupt, and Simone Kriglstein. 2021. What Players Want: Information Needs of Players on Post-Game Visualizations. In CHI Conference on Human Factors in Computing Systems (CHI '21), May 8-13, 2021, Yokohama, Japan. ACM, New York, NY, USA, 13 pages. https://doi.org/10.1145/3411764.3445174

\section{INTRODUCTION}

Video games strongly rely on players being able to make informed gameplay decisions which, in turn, requires appropriate means to convey the necessary information to players. It is thus not surprising that information visualization and video games share a long common past with visualizations taking on many different forms, ranging from simple health bars to more complex representations such as mini maps or technology trees (see, e.g., $[8,54]$ for an overview). Historically, these visualizations were confined to a game's user interface. However, the emergence of online-enabled gaming devices made it possibly to unobtrusively and continuously collect large-scale behavioural data of players, leading to new application areas for data visualization (cf. [46]). Especially within the context of games analytics, visualizations have become an increasingly prominent tool to assist developers in deriving actionable insights from the data [47]. This has lead to a wide variety of visualizations (e.g., $[2,3,14,16,23,48])$ being adapted or specifically developed for gameplay analysis.

While it can be observed that much of the current efforts are directed towards developers - see, for instance, the survey of Wallner and Kriglstein [49] - players have been identified as another important target group $[8,49]$. Players constitute a growing audience, also because of the rise of competitive online gaming and esports which spurred the interest of players in post-play visualizations to 
reflect and improve upon their in-game activities. Indeed, so-called training visualizations have been identified as a primary purpose of visualizations in games besides status visualizations [8, 22]. Training visualizations can be part of the game itself. This can take on the form of graphs showing progression over time such as in StarCraft II (cf. [22]) or visual overlays such as optimal trajectories in racing games (cf. [13]). They can, however, also be provided external to the games, for instance, by websites (e.g., [25, 43]) or stand-alone tools (e.g., $[4,6])$. In academia, however, player-centric visualization is only recently gaining traction with only a few approaches being published to date (e.g., $[1,45])$. We argue that developments in this area are partly thwarted by the fact that there currently exists limited knowledge about which information players rely on to make in-game decisions. Understanding which information is important and why and which goals players pursue with visualizations is, however, critical to create visualizations that satisfy players' needs. Moreover, building an understanding of the information needs of players cannot only contribute to training visualizations but can also be beneficial for developing in-game user interfaces and analysis approaches geared towards developers. Thinking further ahead, our results can also be useful for drawing systematic comparisons between the needs of players and other potential users of visualizations such as spectators.

In an effort to narrow this gap in knowledge we thus conducted a study inquiring into the information needs of players with respect to post-play data visualizations. As information needs can be assumed to vary across games, we focused on different popular genres (realtime strategy, multiplayer online battle arena, and battle royale) in competitive gaming to develop a broader view on the subject. The study consisted of interviewing players $(N=16)$ of games of the above genres to explore and develop themes regarding the aims of using visualizations as well as important information that should be visualized. Subsequently, the identified themes were quantified through an online survey $(N=264)$ to establish the relevance of the themes across the three genres under investigation.

In summary, this paper contributes an overview of the goals players pursue with post-play visualizations along with an inventory of important in-game information and their relevance across genres. Based on our results, we further reflect on a set of high-level recommendations for the design of training visualizations.

\section{RELATED WORK}

Visualizations of in-game data have found wide application within games user research and especially game analytics to handle and make sense of large-scale datasets. The application of visualizations to support analytics has been discussed by several authors (e.g., [15, 31]). Wallner and Kriglstein [49] have charted the landscape of gameplay visualizations, revealing a wide spectrum of different types in use, including spatial (e.g., [15, 48]), temporal (e.g., [2, 38]), and network visualizations (e.g., [28, 33]). While most research efforts in post-play visualizations within game analytics are directed towards analysts, players have been identified as a main audience for visualizations as well $[8,49]$.

Indeed, visualizations have fulfilled an important function in games since the early days of digital gaming by conveying data about the in-game status to players on which they can base their decisions upon (cf. [8]). Bowman et al. [8], using examples from a range of games, have developed a framework for the use of visualizations in games. Similar reviews have also been conducted by Zammitto [54] and Medler and Magerko [32]. They identify a wide array of purposes visualizations can fulfill, ranging from in-game status visualizations to visual representations for communication, progression, and training. Hazzard [22] makes a more high-level classification, distinguishing between status visualizations and training visualizations serving the goal of improving player skill. In this paper, we are concerned with the latter, particularly those who make use of tracked in-game data.

Indeed, training visualizations are garnering increasing popularity, partly fueled by the growth of the competitive gaming scene and more and more developers making in-game data accessible through public APIs. This has resulted in community efforts which utilize the available data to build analytics tools and websites. For instance, OpenDota [43], DOTABUFF [18], and datdota [12] are data platforms for Dota 2 [44] that show various statistics. WOTInspector [25] makes use of heatmaps to depict metrics such as damage or team movement as well as animated maps to visualize data from World of Tanks [52]. Similarly, stand-alone tools such as Scelight [6] for StarCraft II [7] or the WoT-Replay-Analyzer [4] for World of Tanks also heavily rely on visualizations to convey the data. Other tools combine replay functionalities - that enable reviewing matches either directly in the 3D environment (e.g., [42]) or on 2D top-down maps (e.g., $[5,39]$ ) - with different types of visual overlays depicting in-game telemetry data such as trajectories, cone of visions, or kill locations. Usually such tools are game-specific but some such as Shadow [39] also offer a single solution for multiple titles.

In recent years, research has also begun to develop and study player-centric visualizations that utilize telemetry data. Wallner and Kriglstein [50] conducted a comparative evaluation of three visualizations - differing in terms of abstraction level (detailed unit movements vs aggregated troop movement) - for the retrospective analysis of battles in team-based combat games. Results indicated that there was no single visualization that was equally useful for all types of analysis tasks performed by the players. Kuan et al. [27] designed a battle visualization for StarCraft II based on a set of requirements players deemed useful in order to be able to learn strategies. While on overall the system was appreciated by study participants they also pointed out several shortcomings, providing further evidence that satisfying all player demands with a single visualization is challenging.

Research in information visualization has long stressed that understanding user tasks and information needs is crucial for building suitable graphical representations that fulfill them (cf. [37, 51]). However, studies explicitly enquiring into the information needs of players and their goals are still scarce. Karlsson [24] explored how players comprehend information presented to them through ingame visualizations with results suggesting that non-gamers were more attracted to dynamic status information while experienced gamers focused more on high-level overview information such as the mini map. Afonso et al. [1], on the other hand, were interested how information is getting analyzed. Using a spatio-temporal visualization of League of Legends [36] match data, they identified three distinct groups who analyzed the data in different ways (e.g., 
players just describing the information presented, players making speculations based on what is presented).

Lastly, albeit not having training as their primary purpose, visualizations to enhance spectatorship should be mentioned here too as, for instance, watching streams of others people play has been associated with learning as well - although the extent to which this is the case varied across studies (cf. [21, 41]). Anyway, visualizations may provide additional benefits in this regard. For instance, Charleer et al. [11] evaluated two visual dashboards showing realtime match data in addition to the games' user interfaces. Their results indicate that they positively contributed to spectator insight and experience but that careful design is required to alleviate information overload. Kokkinakis et al. [26] developed an app that allows audiences to watch data-driven content alongside esports broadcasts. Evaluations of it at two international Dota 2 tournaments revealed that spectators show propensity to engage with the data-based content. As Charleer et al. [11], finding the right balance in terms of information density was identified as a major challenge. Consequently, it can be expected that our findings can also provide useful pointers for the design of such visualizations.

\section{STUDY DESCRIPTION}

The goal of this research was to explore information needs and to quantify their relevance across genres. We thus opted for a mixedmethods approach to obtain a better understanding about which information players base their decisions on and how this contributes to a) information needs with respect to post-play data visualizations and b) learning and skill development in games. Specifically, we first conducted semi-structured interviews to develop recurring themes which were then quantified through a follow-up online survey.

The whole study was approved by the ethical review board of the Eindhoven University of Technology. Interview guidelines and survey were trialed with two participants each to ensure that the structure and questions asked were appropriate for the intended audience and to pre-test timing and duration of both.

This paper focuses on the first part (a) of this larger study, guided by three research questions. Firstly, understanding information needs requires to understand why players view post-game data visualizations and the goals they pursue with it. As such, our first research question was:

RQ1: Why do players view post-game visualizations or replays?

Creating visualizations that are effective also necessitates to identify which data is important and meaningful for the tasks to be achieved (cf. [37]), addressed by the remaining two research questions. As we are specifically concerned with visualizations for training we were interested in understanding which information players use to inform their gameplay decisions and thus deem important for succeeding in a game, leading to our second research question:

RQ2: Which information do players rely on to make gameplay decisions?

Related to this, we were interested in what information players themselves would include in a graphical representation to help them understand their in-game behaviour and thus perceive as relevant, thereby yielding the following research question:
RQ3: Which information is considered relevant to be included in gameplay visualizations?

Please note that RQ2 focuses on the information players pay attention to during play while RQ3 focuses on data deemed valuable posteriori as we assume that these may be different. For this study we focused on competitive online games, covering three popular genres: real-time strategy (RTS), multiplayer online battle arena (MOBA), and battle royale (BR) games. For the interviews (Section 3.1) we, however, restricted ourselves to one representative game from each genre, selecting games that enjoy high popularity. These games (and genres) were chosen because visual summaries of in-game data are regularly associated with games that rely on skill such as RTS and first-person shooter games [32]. Popular data platforms for these games (e.g., [6, 34, 43]) also suggest a community embracing data-driven solutions for analyzing in-game data. In the following we shortly describe the three games and the general characteristics of the genre:

StarCraft II [7] is a real-time strategy game (RTS) that revolves around three species competing against each other. Starcraft II supports up to four players, with each player controlling a large number of units being led into battle against the opponent(s). It has many of the genre's typical features which include army management, base building, and resource gathering. Players have to manage these aspects in order to emerge victorious. Base building is especially important in StarCraft II to build a strong economy that supports the production of military units. Different RTS games weigh the above features differently and sometimes also include role-playing elements such as character leveling. They are usually played from a top-down, usually isometric, perspective.

Dota 2 [44] is a multiplayer online battle arena (MOBA) game which means that each player controls a single character within a team competing against a second team of players. Each team aims to win the game by destroying the main structure of the enemy. Defensive structures such as towers can be constructed and computer-controlled units are spawned periodically and roam the map. These can be killed for gold which, in turn, can be used to buy items or revive the player character. MOBA games are characterised by a large number of playable characters, each having unique strengths, weaknesses, and abilities. They are usually played on a map from a top-down perspective like RTS games and put strong emphasis on team play and a well-balanced and effective team composition.

PubG [35] is a battle royale game (BR) which involve up to hundreds of players. Each player controls a single character with the goal to eliminate all other players and be the last one alive. Players start with no or limited gear and need to search the environment for equipment and weapons. Every few minutes the map shrinks, resulting in a more confined playable area. Players do not respawn once they have been killed. BR games are usually played in first or third-person perspective and follow in general the above gameplay but often also implement different variations.

\subsection{Part 1 - Interviews}

We first explored our research questions using semi-structured interviews. To recruit a diverse group of interviewees who cover a 
wide range of experiences and views and to ensure that basic criteria were met, we prepared a screener to identify potential interview candidates. The screener asked about demographics, self-reported gaming experience, familiarity with visualizations, as well as for which game (Starcraft II, Dota 2, PubG) they would like to participate. The screener was distributed among relevant subreddits and Discord servers for the selected games as well as the private network of the second author. From 30 responses to the screener we, in the end, selected 16 candidates (15 male, 1 preferred not to reveal their gender) for being interviewed: seven Dota 2, six Starcraft II, and three $P u b G$ players. Age ranged from 20 to 29 (mean $=23.75$, $\mathrm{SD}=2.8)$ with Starcraft II players being slightly older (mean $=25.3$, $\mathrm{SD}=3.3)$ than Dota $2($ mean $=23.6, \mathrm{SD}=1.9)$ and $P u b G($ mean $=21$, $\mathrm{SD}=1.0$ ) players. All of them had multiple years of gaming experience and were familiar with at least different types of charts and diagrams as well as heatmaps. Two participants self-identified as ex-professional Dota 2 players. While one now plays for relaxation only, the other one acts as a role model within the Dota community and serves in captain-like roles. Further two participants were former semi-professional players (one Dota 2 and one Starcraft). Both described themselves now as more casual players but still maintain connections to the community. Of the 16 participants, four highlighted that they are active members of the game community by acting, for example, as commentator, coach, role model, or by running a Discord server. Six participants indicated to the play the games in a more socially oriented way at the time of the interviews while three participants considered themselves highly competitive players. Each of the three games was represented by both social and competitive players. In the following, we will refer to them as $D 1-D 7, S 1-S 6$, and $P 1-P 3$, with the letter indicating the game.

The interviews lasted for about an hour and were conducted via Discord or Microsoft Teams. At the start of the interview participants were introduced to the study's goals and consent to audio record them was obtained. The interview itself was semi-structured and covered the game itself (e.g., why it is played, what they like or dislike about it, which activities they engage with), skill development and learning, decision making, the information they use for that, and the role visualizations play. Recorded interviews were transcribed and analyzed using inductive iterative thematic analysis [9] with respect to our three research questions, that is 1) why players use visualizations of game data or watch replays in order to understand for which purposes they employ them, 2) the information they use to make gameplay decisions, and 3) how relevant they deem different types of information to be visualized. In a first step, the interview transcripts were coded to derive an initial set of themes across all interviews. These were refined based on discussion with a second researcher, followed by re-coding and a second round of discussion to ultimately construct the final themes and higher-level themes. Finally, the interviews were coded according to these. While we interviewed players from different games to approach the topic from different perspectives we paid attention to derive genre-independent categories to increase generalizability. The relative importance for the different genres was assessed using the online survey. The final set of themes together with a short description, the number of mentions, and the number of participants mentioning them, are summarized in Table 1 to Table 3 .

\subsection{Part 2 - Online Survey}

To quantify the themes developed in Part 1 and assess their importance for different genres we administered an online survey through Google Forms. The survey was advertised on subreddits and Discord servers for popular competitive games belonging to the three selected genres, especially for Starcraft II [7], Age of Empires [19], Command \& Conquer [53], League of Legends [36], Dota 2 [44], Fortnite [20], and $P u b G$ [35] as well as some general gaming related subreddits. The survey included basic demographics such as age and gender, previous gaming experience in years, and hours spent playing in the last 30 days prior to taking the survey. Participants also had to indicate the game for which they were taking the survey, with the above mentioned games being available as preset answer options. However, participants could also specify another game. The main part of the survey involved rating the themes inferred through the thematic analysis on 7-point rating scales with labeled endpoints. Generally, one theme was represented by one item in the survey but a small number of themes was split into sub-questions to assess different aspects of them. For instance, KILls $\&$ DEATHS was split to inquiry about their temporal and spatial dimension separately.

In total, we received 292 survey responses of which 15 were excluded because they were filled out with respect to a game not falling withing the three explored genres. This was followed by a longstring analysis to detect careless responders with repeated answering schemes (cf. [30]). This resulted in 13 additional cases to be excluded, yielding a total of 264 responses for analysis. Of those, 249 were male, 10 female, 1 non-binary, and 4 preferred not to disclose their gender. In terms of age, most participants $(=153)$ were in the range of 20 to 29 years, 77 were below 20 years, and 34 were above 29 years. Distribution across the three genres was well balanced with the BR and MOBA genre receiving almost the same number of responses. Specifically, 83 participants responded with respect to BR games (Fortnite $=60, P u b G=23$ ) and 80 with regard to MOBAs (League of Legends $=58$, Dota $2=21$, Heroes of the Storm = 1). The RTS genre received slightly more responses, 101 in total (Starcraft series $=67$, Age of Empires series $=31$, Command $\&$ Conquer series $=3$ ). In terms of gaming experience, participants played games on average since 5.5 years $(\mathrm{SD}=4.3, \mathrm{~min}=<1$ year, $\max =20$ years $)$ and played on average 63.6 hours $(\mathrm{SD}=54.2$, $\min$ $=0, \max =350)$ in the 30 days prior to taking the study.

Likert-scale responses to the survey were treated as ordinal and thus analyzed using non-parametric tests. In order to assess differences between the three investigated genres we calculated Kruskal-Wallis tests for each category (i.e. question). Due to the number of comparisons we calculated adjusted p-values for these tests to control for false discovery rate (Benjamin-Hochberg procedure). If the test was significant, it was followed by Dunn's post-hoc test with a Bonferroni corrected alpha level of .016. Figure 1 shows the results with respect to why players rely on post-game data visualizations or watch replays. Figure 2 depicts which type of information about themselves or the opponents players find useful for making gameplay decisions. Finally, Figure 3 examines how relevant players deem the inclusion of certain information in a visualization. 
Table 1: Themes regarding the reasons for watching replays and viewing post-game data visualizations and summaries, along with the number of participants mentioning it (\#P) and the number of statements (\#S).

\begin{tabular}{|c|c|c|c|c|c|}
\hline Theme/Category & Description & $\# \mathbf{P}$ & & $\# \mathbf{S}$ & \\
\hline IMPACT OF DECISIONS & $\begin{array}{l}\text { insights on the effects of decisions and their influence on the game, if they were the } \\
\text { right decisions or whether others would have been better }\end{array}$ & m & 8 & & 12 \\
\hline EFFICIENCY & improving efficiency with respect to & & & & \\
\hline ResourCES \& ITEMS & $\begin{array}{l}\text { gaining insights on the efficient use of resources and items, including the timing } \\
\text { of use as well as the optimal choice of items and on what to spend resources on }\end{array}$ & $\mathbf{E}$ & 9 & & 12 \\
\hline SEQUENCING & $\begin{array}{l}\text { gaining insights with regard to sequencing of items, buildings, levels \& upgrades, } \\
\text { etc. }\end{array}$ & - & 3 & $\boldsymbol{\square}$ & 5 \\
\hline MOVEMENT & movements and how important it is & - & 3 & $\mathbf{\square}$ & 3 \\
\hline UNDERSTAND SITUATIONS & viewing of specific situations that the player did not understand or was surprised by & $\mathbf{0}$ & 7 & ש & 7 \\
\hline Predictability & being able to predict and disguise behaviour & & & & \\
\hline BEING UNFORESEEABLE & to understand how to be less predictable for others & $\mathbf{\square}$ & 5 & $\mathbf{u}$ & 7 \\
\hline ANTICIPATE ENEMY & to be able to better anticipate and predict enemy plans and actions & $\mathbf{\square}$ & 4 & $\mathbf{\square}$ & 6 \\
\hline LEARN FROM OTHERS & $\begin{array}{l}\text { watching how other people play to understand how well they do and what actions } \\
\text { they took as well as identifying new ways of playing }\end{array}$ & $\mathbf{n}$ & 6 & & 7 \\
\hline LEARNING & $\begin{array}{l}\text { identifying learning points and mistakes, gaining insights in how much different } \\
\text { factors influence the game, and picking what to focus on to improve next }\end{array}$ & $\square$ & 5 & $\mathbf{\square}$ & 6 \\
\hline Personal SATisfaction & viewing points, score, gold, damage dealt, etc. for personal satisfaction & I & 2 & I & 2 \\
\hline
\end{tabular}

\section{RESULTS}

In the following we discuss our results based on the three research questions outlined in Section 3. For the sake of presentation, we will designate median scores $<4$ as low, $\geq 6$ as high, and as moderate otherwise. Detailed statistics can be found in Figure 1 to Figure 3. Categories derived from the thematic analysis of the interview transcripts are written in small caps.

\subsection{Why do players view post-game visualizations or replays (RQ1)}

All except one category (cf. Table 1) fall under the larger umbrella of players wanting to understand and improve upon gameplay, showing the high relevance of post-play visualizations for training and learning purposes. Interviewees commented about replays and visualizations being valuable for LEARNING about the game in general, especially also by being able to LEARN FROM OTHERs by observing how they play, what mistakes they make, and potentially identifying new ways of playing. Interviewees expressed interest to review the ImPACT of Decisions to get an impression how their choices influenced the game. Participants also appreciated replays and visualizations for UNDERSTANDING SitUATIONS which were not obvious during play. This is nicely captured by the following comment from S3: Sometimes you lose one, and if I don't get why I lost, I check the replay and try to figure that out. These aspects were all rated highly relevant in the survey across all three genres (see Figure 1), confirming the impressions gained from the qualitative analysis.

Players were also especially concerned with improving their EFFICIENCY within a game by deducing ways to better use RESOURCES \& ITEMS; to learn about SEQUENCING, that is, in which order and when to use upgrades or construct buildings; and to improve Movement, including the positioning of units. The following quote exemplifies the general importance of efficiency well:
I would like to see when I could have done something faster or more efficient [S4]. While MovEMENT was considered relevant for all three genres, SEQUENCING was rated significantly more relevant in case of RTSs and BRs compared to MOBAs, while REsources \& ITEMS showed significant differences between RTSs and MOBAs.

Another frequent reason for referring to visualizations and replays was players' desire to better Anticipate EnEMY behavior and thus being able to predict future actions while at the same time also gaining insights on how to disguise their own undertakings to be more unforeseeable. For example, the following comment by P1 reflects on the former aspect:

Especially the death cam is important. It tells you how and why you were vulnerable but also gives insight in how the enemy killed you, so you can try to learn from their thinking and technique.

Learning to BE UNFORESEEABLE was especially considered useful in the case of BR games but was only considered moderately relevant in case of MOBAs and RTSs. Being able to anticipate the enemy was rated more relevant in case of RTSs and BRs than for MOBAs.

Lastly, interviewees made remarks about how watching replays can serve as Personal Satisfaction by seeing how well oneself succeeded or others were failing, but those were few. This was also evident in the survey responses were participants rated it of lower relevance compared to all the other aspects related to learning about different aspects of a game.

\subsection{Which information do players rely on to inform gameplay decisions (RQ2)}

Table 2 lists the different types of information players considered necessary for making informed gameplay decisions. Participants concluded that both information about oneself (or the team one is being part of) and about the opponent(s) are important for making gameplay decisions. 

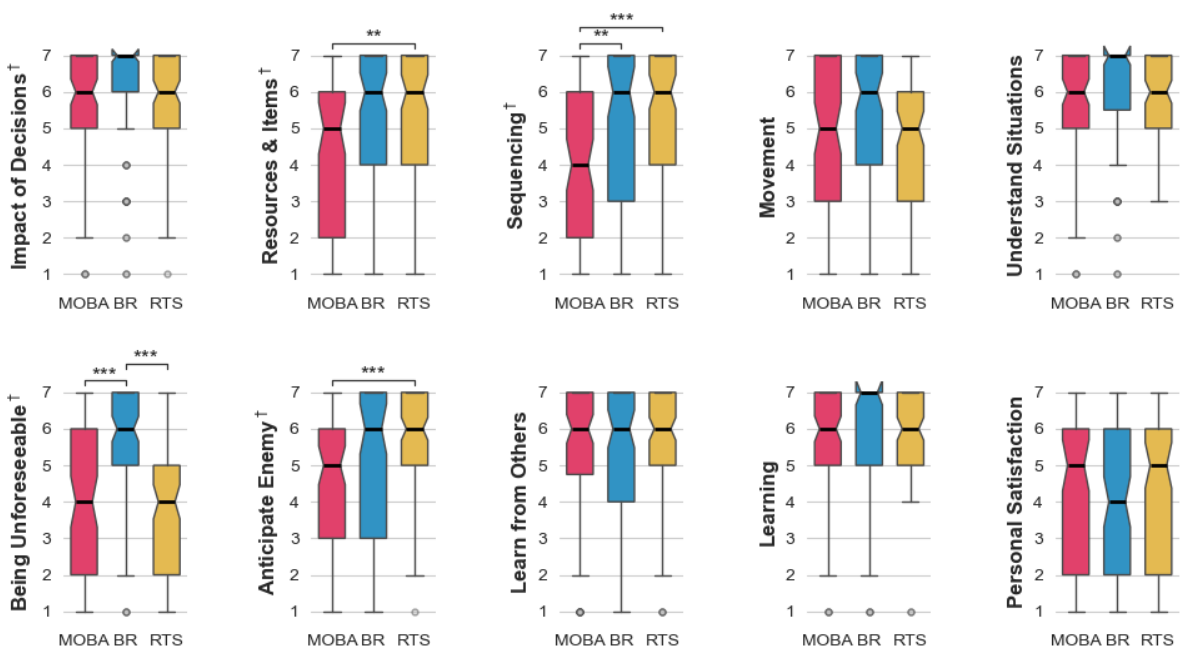

${ }^{\dagger}$ Kruskal-Wallis $\mathrm{H}$ test significant at false discovery rate adjusted $p<.05$, Dunn’s post-hoc test for multiple comparisons significant at ${ }^{*} \alpha=.016$ (Bonferroni corrected), ${ }^{* *} \alpha=.01,{ }^{* * *} \alpha=.001$

Figure 1: Reasons for watching replays or viewing post-game data visualizations $(1=$ not relevant, $7=$ very relevant $)$ across the different genres.

In general, players considered the same type of data important to know about both sides. Knowing the Positions \& Movement about oneself (and one's team) as well as about the enemy was the most mentioned type of information to influence decisions. D3, for instance, noted that generally, enemy movement is very important combined with your own position in the game. D7 elaborated further on this, saying:

See where people are walking, and deduce what their plans are. Figure out their goal, what and why they want to achieve. Then anticipate if/how you can stop this $[\ldots]$

This also showed in the ratings, with participants rating it of highest importance across all genres (cf. Figure 2).

Players also made frequent references about how ENTITY CHARACTERISTICS ${ }^{1}$ and their specific LEVELLING influence the choices they make. While both categories are about playable entities we distinguished between the two, to highlight that players consider both the static characteristics that entities possess while also being influenced by their evolving attributes through continual leveling. For example, D1 commented:

Heroes that I know are good against me influence me mostly. When I play Juggernaut [a hero in Dota 2], for example, I know there are only a few heroes that can really damage me. Based on that, I can decide where I am safe or what I can reasonably win.

Both showed to be most relevant in MOBAs and RTSs, with players of BR games only attaching low (maximal moderate) relevance to it. Related to this, participants reflected on how their EQUIPMENT (e.g., weapons, items) and that of the opponent has implications

${ }^{1}$ Entity is used here as a generic term for all different types of playable characters (e.g., units, heroes, race) within a game. on their in-game interactions, as illustrated well by the following comment from D1:

Other than that, obviously your items, gold, and weapons are important; those decide your options and what lines of play you have available.

While generally considered of moderate to high importance, ratings showed significant differences between the genres, with respondents considering it more important for MOBAs and BRs than for the RTS genre. The RESOURCES one or the contender(s) have at their disposal were also mentioned as having an effect on the decisions being made. Resources were considered as having moderate to high importance in all the genres with differences in the ratings (except one) being statistically insignificant. Lastly, interviewees elaborated - although rarely - on how the role of the player or the roles in the opponent's team affects their choices. The quantitative results also only attested high relevance to it in case of MOBAs, with scores for RTS and BR games (low to moderate) being significantly lower.

How information provided through the CHAT influences players was only mentioned with reference to the own team which is reasonable because the chat of the other team is not accessible during gameplay. For example, D7 noted:

What your teammates are saying is also very important; you can't see everything yourself, mostly there's someone coordinating (like me) who provides information about what to watch out for, and where the enemy is moving and what we should do.

The communication between team members was considered a highly important source of information in case of MOBA and BR games, while it was considered of significantly less (albeit still moderate) relevance for RTS games.

It is important to highlight that throughout the interviews participants focused more on which opponent-related information they 
Table 2: Categories of which information is important when making decisions during play, along with the number of participants mentioning it (\#P) and the number of statements $(\# \mathrm{~S})$.

\begin{tabular}{|c|c|c|c|c|c|}
\hline Theme/Category & Description & \#P & & $\# \mathbf{S}$ & \\
\hline OPPONENT & information important to know about the opponent & & & & \\
\hline POSITIONS \& MOVEMENT & the importance of knowing enemy movement and positioning & - & 14 & & 19 \\
\hline BUILDINGS & information about enemy buildings and its importance for making decisions & $\mathbf{\square}$ & 4 & & 13 \\
\hline Play Style & how the enemy playstyle (e.g., aggressive, defensive) influences one own's playstyle & ש & 8 & & 12 \\
\hline ENTITY* CHARACTERISTICS & $\begin{array}{l}\text { unique and specific characteristics about the race, hero, or build the enemy is playing } \\
\text { and how it influences decision making }\end{array}$ & ש & 6 & & 7 \\
\hline EQUIPMENT & $\begin{array}{l}\text { enemy equipment, weapons, items, abilities, or similar that provide options and } \\
\text { influence play }\end{array}$ & $\mathbf{\square}$ & 4 & ש & 6 \\
\hline LEVELS & the importance of levels of enemy units, heroes, buildings etc. for decision making & घ & 3 & - & 3 \\
\hline RESOURCES & how the resources the enemy has available influences decisions & 匹 & 3 & घ & 3 \\
\hline HIT PoINTs & how enemy hit points or health influences decision making & 【 & 2 & I & 2 \\
\hline Kills \& DeAThs & how enemy kills and deaths influence decisions & 】 & 2 & 】 & 2 \\
\hline StART LOCATION & how the enemy starting location can influence decision making & $\mathbf{I}$ & 1 & I & 1 \\
\hline TEAM ROLE & how the roles in the enemy team influences decision making & $\mathbf{I}$ & 1 & I & 1 \\
\hline MYSELF \& ONE'S TEAM & information important to know about the player (or the player's team) & & & & \\
\hline POSITIONS \& MOVEMENT & $\begin{array}{l}\text { how the player's and/or team's positioning and movement influences decisions and } \\
\text { how to proceed }\end{array}$ & & 7 & & 8 \\
\hline СНАТ & how information provided through the team chat is important for decision making & $\square$ & 5 & $\mathbf{\square}$ & 5 \\
\hline ENTITY* CHARACTERISTICS & $\begin{array}{l}\text { the importance and influence of the chosen hero, race, build, and units (and their } \\
\text { specific characteristics) on play and how decisions are made }\end{array}$ & $\mathbf{\square}$ & 4 & $\mathbf{\square}$ & 4 \\
\hline EQUIPMENT & $\begin{array}{l}\text { how the player's or team members' equipment, abilities, weapons, and items influence } \\
\text { play }\end{array}$ & 匹 & 3 & - & 3 \\
\hline RESOURCES & the importance of the player's and/or the team's resources on decision making & 】 & 2 & I & 2 \\
\hline LEVELS & how levels of, e.g., buildings and abilities decide which decisions are made & - & 3 & घ & 3 \\
\hline TEAM ROLE & how the role of the player in the team influences the plan and actions to be taken & 匹 & 3 & - & 3 \\
\hline
\end{tabular}

${ }^{*}$ Note: Entity is being used as a generic term for different playable characters such as heroes, units, and more high-level constructs which can have unique characteristics as well. Categories written in italics have been mentioned for oneself and the opponent.

rely on to inform their decisions. Especially, knowledge about the enemy's BuILDINGs was a reoccurring theme, particularly in case of RTS players as it is considered important for taking appropriate counter measures or for deciding when to attack. Survey responses confirmed the high relevance in case of RTSs but also showed that it can be moderately valuable for making decisions in MOBAs and BRs. Players also indicated to pay attention to the Hit Points (i.e. the amount of damage a character can withstand) or health the enemy has, with players indicating that this, for example, decides whether to engage or retreat. While not mentioned often in the interviews, survey respondents confirmed its high importance for MOBAs and BRs but deemed it only of moderate relevance for RTS games. StART LocAtions as well as locations of Kills \& DEATHS caused by and of the enemy were also noted, albeit very rarely. The quantitative results confirmed this, with both being rated to be only of low to moderate importance, with the KILLS \& DEATHS in case of MOBAs rated highest. Lastly, interviewees made frequent references to the general Playstyle of the opponent, for instance, if the enemy follows a defensive are aggressive strategy or shows signs of greediness. Playstyle also formed, together with Positions \& Movement and Resources, the most highly rated category independent of genre.

\subsection{Which information is considered relevant to be included in gameplay visualizations (RQ3)}

Asked about which information players judge relevant to be included in a post-game visualization, interviewees made recurring references to the visualization of specific data as-is (e.g., kills, scores, use of resources) but also discussed the relevance of visualizing higher-level knowledge (e.g., map control, fights, being in advantage) that requires the integration and combined analysis of various low-level data (e.g., individual positions, death locations, character levels). Table 3 summarizes the major themes arising from the interviews with high-level concepts written in boldface. Figure 3 presents the scores for the different categories across the three genres.

Some of the emerged categories relate directly to the information players rely on during gameplay (in particular Movement, Resources, Kills \& Deaths, and Build Order). As during play, information about MOVEMENT and Resources proved to be the most discussed aspects to be visualized, reinforcing their importance for succeeding in the investigated games/genres. Information on movement about oneself or the opponent was rated of moderate to high importance with no significant differences between the genres. Resources although also scoring in the range from moderate to high 

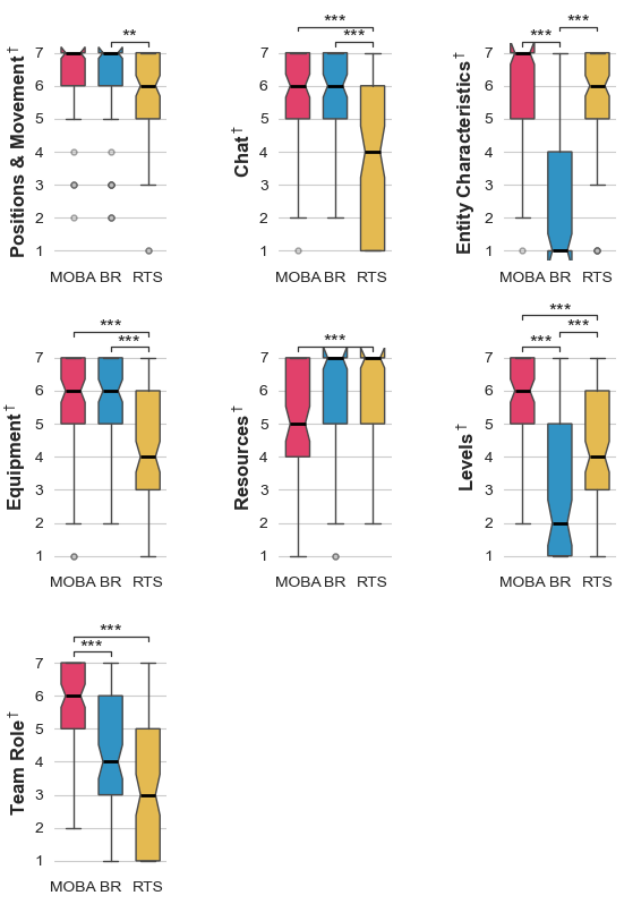

MOBABR RTS
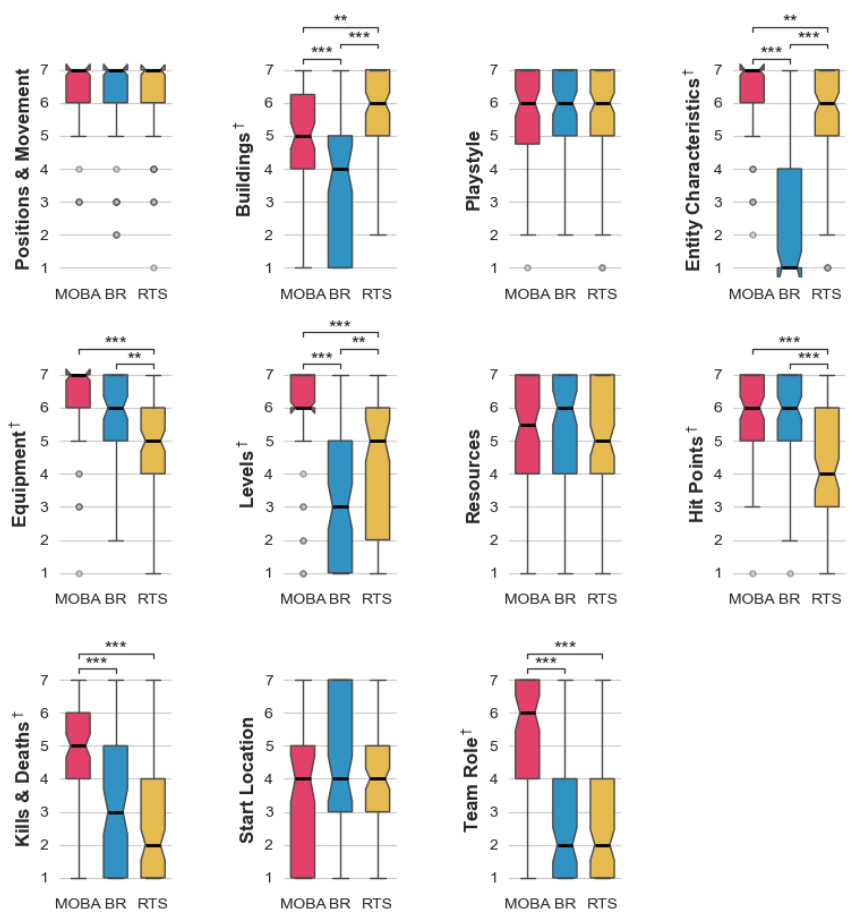

$\dagger$ Kruskal-Wallis $\mathrm{H}$ test significant at false discovery rate adjusted $p<.05$, Dunn’s post-hoc test for multiple comparisons significant at ${ }^{*} \alpha=.016$ (Bonferroni corrected), ${ }^{* *} \alpha=.01,{ }^{* * *} \alpha=.001$

Figure 2: Importance $(1$ = very unimportant, 7 = very important) of different types of information about the player itself or the own team (left) and about the enemy (right) in order to make gameplay decisions across the different genres.

were, however, considered of significantly higher relevance in RTS games compared to BRs and MOBAs.

Interviewees also suggested to include details about ITEM USE and about general ScoREs such as points, position in the leaderboard, and similar to see how well one is doing in the game (also in relation to others). Both, however, were considered of moderate to low importance with scores also varying significantly across the genres. KILLS \& DEATHS (both in terms of location and time) and the Number of Units were also found worth visualizing with the former being moderately important for BR and MOBA games but significantly less so for RTSs whereas the latter showed the opposite pattern, being considered highly important for RTSs but unimportant for MOBAs and BRs. Information about Levels $\mho$ ABILITIEs was considered of moderate importance for RTS and MOBA games with both showing significant differences to BRs for which it was considered irrelevant. In contrast, the START LOCATION was of high importance for BRs with significant differences to RTSs and MOBAs (very low relevance). Visualization of BUILD ORDERS was considered highly relevant for RTS but significantly less so for the other two genres. DAMAGE dealt or received emerged as another important source of information, being most important for BRs, followed by MOBAs, and lastly RTSs with significant differences between the scores. Players also reflected upon the value of visualizing the Field of VIEW in order to be able to assess which areas were observed or not, potentially causing them to miss important information. It was rated of moderate to high importance (for BRs) but the differences were not significant.

In addition to players reflecting upon the visualization of data items, the interviews also revealed an interest in visualizations that not only show the data 'as is' but which rather show higher-level knowledge extracted from the data. For instance, S6 reflected on how visualizations can help to draw attention to mistakes made:

Yeah with pure data, you basically ask the gamer to make conclusions by themselves, I think that is the bottleneck. I think analyzing data and drawing mistakes from patterns, can really help. It's about highlighting the big mistakes. That is hard though, since there are so many elements contributing to the big picture.

Among those, the most recurring concept in the interviews was about conveying details about FigHTs and how they influenced a game. Fights were also considered highly relevant by the survey respondents across all genres, of which MOBA players rated it the highest. Related to this, the interviews revealed a tendency to be able to observe who controls which parts of the map during which time periods and how the front line changes, i.e. MAP ConTrol. The ratings confirmed the high importance of this kind of information for MOBA and RTS players. D1 mused about how current visualizations lack in this aspect:

However, even with all the visualizations used in coverage of professional matches, I have never seen something like that, using front line, zone of control, map 
Table 3: Themes with respect to the visualization of data for post-play analysis. Categories in boldface denote higher-level concepts requiring the integration of several data types. \#P indicates the number of participants and \#S the number of statements.

\begin{tabular}{|c|c|c|c|c|c|}
\hline Theme/Category & Description & $\# \mathbf{P}$ & & $\# \mathbf{S}$ & \\
\hline Movement & $\begin{array}{l}\text { visualizing the movement of heroes and units across the map, also in relation to } \\
\text { other relevant statistics }\end{array}$ & ש & 9 & & 20 \\
\hline Resources & $\begin{array}{l}\text { visualizing acquisition and use of resources, also in relation to other statistics and } \\
\text { events, to gain insights regarding their optimal use }\end{array}$ & 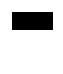 & 9 & & 20 \\
\hline Fights & $\begin{array}{l}\text { times when important fights happened, specifically combined with other relevant } \\
\text { statistics to be able to see how these influence a game }\end{array}$ & $\mathbf{\square}$ & 4 & - & 8 \\
\hline ITEM USE & $\begin{array}{l}\text { suggestions to visualize the timing of item use, also in relation to other statistics and } \\
\text { events }\end{array}$ & a & 5 & & 8 \\
\hline SCORES & $\begin{array}{l}\text { visualizing scores, points, rankings, or similar to be able to see who is ahead at which } \\
\text { moment, why, and how this relates to other statistics }\end{array}$ & E & 7 & & 8 \\
\hline MAP CONTROL & $\begin{array}{l}\text { suggestions on visualizing map control, i.e. which team controls which parts of the } \\
\text { map over time, and where the front line is }\end{array}$ & $\mathbf{\square}$ & 4 & 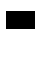 & 7 \\
\hline KiLLS ひ Deaths & visualizing kills and deaths and their impact on the game & $\mathbf{\square}$ & 4 & & 7 \\
\hline Blocking of SuPPlies & $\begin{array}{l}\text { observing time spans when resources are being cut off, to see how this impacts the } \\
\text { game and how long it takes to recover from it }\end{array}$ & - & 3 & $\mathbf{\square}$ & 5 \\
\hline Number of Units & army size and/or the number of workers & - & 3 & $\mathbf{\square}$ & 5 \\
\hline LeVELS $\mho$ ABilities & $\begin{array}{l}\text { seeing when leveling/upgrading is initiated and finished and when abilities are } \\
\text { available, when they are used and when they are available again }\end{array}$ & - & 3 & - & 5 \\
\hline START LOCATION & $\begin{array}{l}\text { viewing the enemies' starting location(s) in relation to other statistics (e.g., win rate) } \\
\text { to understand how this affects the game }\end{array}$ & - & 3 & ש & 5 \\
\hline Pressure & $\begin{array}{l}\text { suggestions to quantify, process, and visualize the amount of pressure applied to and } \\
\text { by an enemy to gain insights in how this influences games and winrates }\end{array}$ & - & 3 & - & 5 \\
\hline COMPARISON & $\begin{array}{l}\text { visually comparing one's own game, to create reference and give statistics a semantic } \\
\text { meaning }\end{array}$ & & & & \\
\hline Player Base & $\begin{array}{l}\text { to compare oneself with how other players perform on average in relation to } \\
\text { specific aspects }\end{array}$ & $\mathbf{\square}$ & 4 & ש & 5 \\
\hline Personal Progress & to track the improvement with respect to one's own progress and learning curve & 】 & 2 & 匹 & 3 \\
\hline OPTIMUM OR GoAL & to compare to a set goal or the optimal way of playing, sequence, etc. & $\mathbf{I}$ & 1 & $\mathbf{I}$ & 1 \\
\hline Match Fairness & $\begin{array}{l}\text { suggestions for quantifying and visualizing the fairness of a match, to help understand } \\
\text { reasons for winning and losing and help people to be at peace with losing }\end{array}$ & 】 & 2 & $\mathbf{\square}$ & 4 \\
\hline BUILD ORDER & order and timing of constructing buildings & - & 3 & $\boldsymbol{\square}$ & 4 \\
\hline DAMAGE & the amount of damage dealt to or by specific characters, units, or teams & - & 3 & - & 4 \\
\hline FIELD OF VIEW & $\begin{array}{l}\text { suggestions to visualize how the own's and enemy's field of view moved to be able to } \\
\text { gain insights regarding efficiency as well as which information was missed because } \\
\text { one did not observe a specific area of interest at the right time }\end{array}$ & - & 3 & - & 4 \\
\hline Being in Advantage & $\begin{array}{l}\text { processing and visualizing moments where one team or player has a distinct advan- } \\
\text { tage over the other(s), if this is noticed and if it is utilized properly }\end{array}$ & $\mathbf{I}$ & 1 & 匹 & 3 \\
\hline
\end{tabular}

control, etc. you have to translate that from the map yourself, a little. Visualizing this could really provide great insights - I think.

Related to this, players also considered it relevant to understand the Blocking of Supplies and the consequences arising from it, with RTS players considering it highly relevant. Differences to MOBA and BR players who rated it of moderate and low importance, respectively, are significant. The concept of PrEssure also arose repeatedly in the interviews. Players commented on how important it is to apply pressure to the enemy and how they would appreciate a way to quantify and visually convey the amount of pressure. Survey ratings also attested moderate to high importance to it with no significant differences between the genres.

Two participants also made suggestions for visualizing the МATсH FAIRNESS to help understand why a match might have been lost or won. Survey respondents thought it to be of moderate relevance in all three genres, with no significant differences between them. Showing if someone has a distinct advantage (BEING IN AdvantAGE) during a match - although only suggested by one participant to see, for instance, if the advantage was used or missed - was considered of high importance for all three genres by the survey respondents. Lastly participants made recurring statements specifically about visualizations that would allow them to make COMPARISONS to situate their performance either (in order of decreasing number of mentions) with respect to the general Player BAsE, their Personal Progress, or to an Optimum or GoAl. Despite the varying relevance in the interviews, survey respondents agreed that all three types of comparisons are valuable (moderate to high) for post-play analysis with no pronounced differences between the genres. 

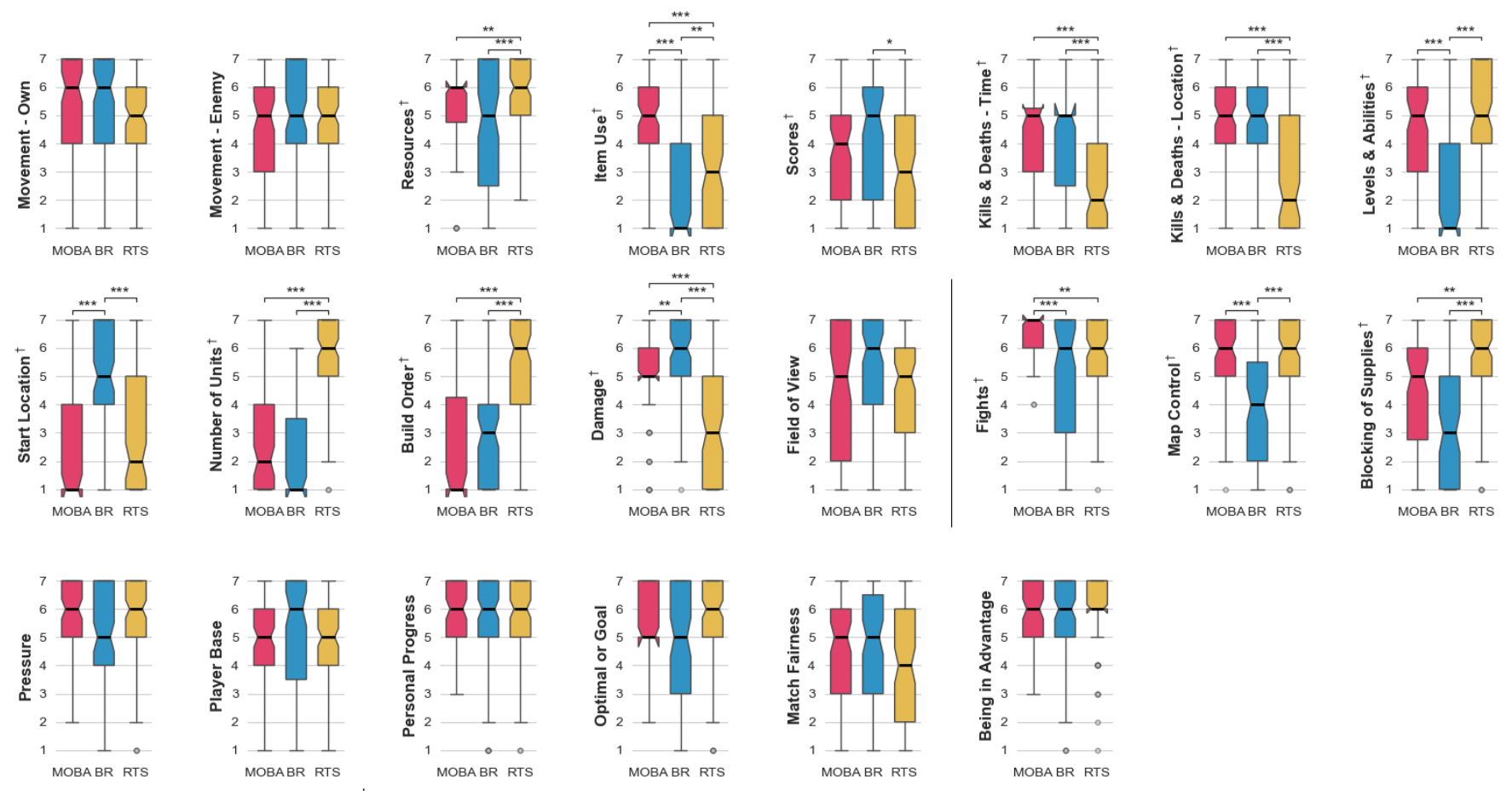

$\dagger$ Kruskal-Wallis $\mathrm{H}$ test significant at false discovery rate adjusted $p<.05$, Dunn's post-hoc test for multiple comparisons significant at ${ }^{*} \alpha=.016$ (Bonferroni corrected), ${ }^{* *} \alpha=.01,{ }^{* * *} \alpha=.001$

Figure 3: Relevance $(1=$ not relevant, 7 = very relevant) of visualizing certain types of information for gaining new insights, learning, and/or being more motivated and confident across the different genres.

\section{DISCUSSION}

Our results provide an overview of which goals players pursue with post-play visualizations (cf. Table 1), which information is relevant for them for decision making (cf. Table 2), which information to visualize (cf. Table 3), and how their importance varies across three genres (see Figure 1 to Figure 3).

The findings show that players of competitive games do have an interest in using post-play visualizations of in-game data as well as replays to help them learn about a game and develop new skills. Viewing them for personal satisfaction, on the other hand, was of comparatively lower relevance. Learning has also been shown to be a reason for watching streams [21] but Sjöblom and Hamari [41] found that learning about game strategies is of subordinate importance, hypothesizing that the live aspect could be detrimental in this regard. Visualizations are different in this sense as they can be viewed and manipulated by the users themselves, enabling learning about one's own specific interests and at one's own pace. While players valued visualizations to learn from their behaviour and from others in general, our analysis also revealed more specific themes revolving around situational understanding, awareness of the consequences of decisions, a strong focus on improving efficiency in various aspects, as well as predictability. While most reasons were common across all three genres, some also varied across them (cf. Figure 1). This shows the importance of tailoring training visualizations to the specific and also varying information demands of players. For example, a visualization to convey the impact of decisions will need different design considerations than a visualization aiming to help improve efficiency.

As with the reasons for using visualizations, certain information players rely on during play and would like to see visualized was considered equally important while the relevance of other information varied across genres. This can partly be explained by some aspects being highly game specific (e.g., build orders to quickly establish a strong economy are quite specific to RTS games) and partly by the number of units involved as well as the importance of individual unit characteristics and the level of cooperation needed between players. For example, players of RTS games need to command a large number of units where individual unit characteristics take on less importance while MOBAs put strong emphasis on character attributes and leveling of the heroes which also have to complement each other well. This places different emphasis on the level-of-detail of the data to be displayed.

\subsection{Implications for the Design of Post-Game Visualizations}

In the following we reflect on high-level implications for the design of post-game visualizations, in particular visualizations for training, skill development, and learning as these formed the main reasons for using such visualizations.

5.1.1 Careful choice of data. With respect to the data players rely on during play (see Table 2), it is evident that, as mentioned above, 
some data has general importance across all three genres (cf. Figure 2). The most relevant being information on movement which can be attributed to the highly spatial nature of the games under investigation. However, other information varied significantly across the genres which is to be expected as their gameplay mechanics are different. This places emphasis on deliberately choosing the data to be included in a visualization in order to keep it simple, avoid visual clutter, and to make it useful for players of the respective games. As with visualizations part of a game's user interface [10] - or visualizations in general for that matter - post-play visualizations need to carefully consider the information they present to neither overload the player with nor miss out on important information. Our results provide guideposts on which aspects to focus on for the different genres (cf. Figure 3).

5.1.2 Choice of visual representation. Once the data has been selected appropriate representations for the data have to be chosen, which is influenced by the data to be presented and the task to be supported. The goals summarized in Table 1 and relevant information for players listed in Table 2 and Table 3 can be helpful in this respect. For example, a visualization focused on efficiency may put stronger emphasis on the temporal aspects of the data while a representation of map control may attach greater weight to spatial relationships. Besides, our results can be valuable for choosing the appropriate level of detail for representing the data (i.e. individual or aggregated). Revisiting the RTS example above, the lower importance of individual unit characteristics paired with the large number of units may make, for instance, aggregation of movement data an acceptable option.

Lastly, while we did not systematically code for it, we would like to - also because we feel it received comparable less attention raise another issue which surfaced anecdotally in the interviews, namely that the represented information needs to be reliable. This is, for example, reflected in the quote of S6 in Section 4.3 who raises the point that making inferences oneself can be prone to errors or in the following statement from P1: I also question how realistic it is in how data is interpreted. Indeed, Wallner et al. [48], proposing a developer-centric visualization, found that game developers started to question the accuracy of the evaluated visualization when the depicted data did not match the player behaviour they expected. In short, players need to have confidence that the displayed data is represented in a way that is trustworthy (see [29] for an overview of the different dimensions of trust in information visualization) and that they can rely on it to make correct inferences with respect to the goals of the analysis.

5.1.3 Take advantage of data not accessible during gameplay. In general, players discussed information about themselves (or the team they are part of) and information about their opponent(s) with the latter receiving slightly more attention. When reflecting on decision making players may implicitly assume certain information as given as they are constantly aware of it during gameplay. As such they thus might not have explicitly stated it which could partially explain why information about oneself resulted in fewer categories than about the enemy. While there is a large overlap between the themes, some where unique to either of them. Some of the differences may also stem from the fact that not all information is accessible to players during play such as the chat of the enemy team. Post-game visualizations are, however, not restricted to the information visible during play and should take advantage of being able to include data otherwise inaccessible. This can increase situational understanding and benefit the assessment of the impact of decisions (which have been shown to be important reasons for reverting to visualizations and replays) because cause-effect relations between the own and enemy behaviour can be made apparent.

5.1.4 Information becomes more/less relevant in retrospect. Our analysis also showed an overlap of themes between the information players rely on during gameplay and they deem relevant to be included in visualizations, which is to be expected. However, it also hinted at the fact that the relevance of certain information changes. For instance, enemy movement was considered more relevant while playing whereas the movement of the player itself gained more relevance in post-play visualizations (compare Figure 2 with Figure 3). That is, post-game visualizations should consider the fact that certain information may become more important retrospectively.

5.1.5 Convey high-level concepts. In terms of the type of information players consider relevant, the emerged themes not only showed on interest in specific 'low-level' data (e.g., number of units, kills and death locations) but also in 'high-level' concepts (e.g., pressure, map control, fights) which require the integration of different data types. For example, fights are not only characterized by the individual death locations but also who shot at whom, from where units joined a fight and where they retreated to, and similar. Our results indicate that these high-level concepts were generally rated highly relevant across all genres, while the value of different types of individual data varied strongly between them. That is, training visualizations should move beyond visualizing individual metrics but rather combine them in a way that conveys high-level knowledge that relates to macro gameplay concepts. This is not to say, however, that such visualizations should completely neglect the representation of low-level data. Rather, it should be seen as an opportunity to carefully combine both aspects to help players understand how a series of small decisions impacts gameplay on a higher level. In that sense, such visualizations could benefit by including artificial intelligence and data analytics methods to process and extract these high-level information from the individually tracked in-game metrics.

\subsection{Limitations \& Future Work}

Our study was exploratory in nature involving a large number of statistical tests. While we took measures to control for the number of comparisons the results should still be regarded as exploratory rather than confirmatory. In this sense, significant results should be interpreted as cases where visualization designers should be particularly mindful of different game genres having different information needs and priorities.

In our analysis we focused on three popular genres to cover a variety of gameplay mechanics occurring in competitive games. While it can be expected that our results, at least partly, transfer to others genres as well (e.g., first-person shooters), future work needs to confirm our results across further genres. Our focus on three genres also means that the list of goals and relevant information for players may not be exhaustive. In that sense, further studies with 
other games will help to build a more comprehensive inventory of player goals and information needs.

Different user groups with their different interests and requirements (e.g., developers using analytics to improve game design and balancing, players to improve performance, spectators in being able to effectively follow gameplay) will also likely have varying information needs. However, not much systematic knowledge about the needs of the different groups currently exists. As such, our study can contribute and be useful for understanding differences between different types of users by conducting similar studies with other user groups in the future.

In this paper we focused on the information players would like to see rather than on the specifics characteristics of the data, for instance, if the data is spatial or temporal and which specific visualization techniques should be used to visualize it. This was a deliberate choice as the specific attributes of the information can differ from game to game. However, multiple taxonomies for choosing appropriate visual representations for specific data types have been proposed in information visualization (e.g., [40]), games (e.g., [49]), and related fields such as competitive sports (e.g., [17]) to date. These taxonomies can be useful in our context as well and hence we considered it out of scope of this work.

With many analytics tools being already available to players (see Section 2), an interesting avenue for future research would be to evaluate existing analytics tools with respect to the information needs and design implications suggested in this paper. While some of the identified differences in genres can be explained by the different genre-specific gameplay mechanics other differences may require further inquiries to better understand why certain information is more or less useful. Future work can also build upon our results to build new post-play visualizations, specifically training visualizations, that align with players' goals and information needs. Evaluating these will bring along new challenges since assessing training progress and skill development will require long-term studies that are carefully controlled for confounding factors.

\section{CONCLUSIONS}

Visualizations for the respective analysis of gameplay have gained increased interest among players, especially those that are interested in competitive gaming. However, little is known about the specific aims players pursue by reviewing such visualizations and which information is deemed relevant for them to be included. Our study has shown that post-game visualizations are mostly reviewed for training purposes, compared to replays where learning is of subordinate importance [41]. By comparing information demands across three popular genres we established an inventory of relevant information for them. Paired with the specific analysis goals and the high-level implications arising from our analysis this can serve as a practical reference for the design of goal-directed and meaningful post-play visualizations.

Future work may, however, investigate other genres to expand upon this initial inventory. With player-centric visualization still being a new research area, we hope our findings spark inspiration for creating visualizations that address tasks not yet or only marginally covered by existing gameplay visualizations.

\section{REFERENCES}

[1] Ana Paula Afonso, Maria Beatriz Carmo, Tiago Gonçalves, and Pedro Vieira. 2019. VisuaLeague: Player performance analysis using spatial-temporal data. Multimedia Tools and Applications 78 (2019), 33069-33090. https://doi.org/10. 1007/s11042-019-07952-z

[2] Shivam Agarwal, Günter Wallner, and Fabian Beck. 2020. Bombalytics: Visualization of Competition and Collaboration Strategies of Players in a Bomb Laying Game. Computer Graphics Forum 39, 3 (2020), 89-100. https://doi.org/10.1111/ cgf. 13965

[3] Sabbir Ahmad, Andy Bryant, Erica Kleinman, Zhaoqing Teng, Truong-Huy D. Nguyen, and Magy Seif El-Nasr. 2019. Modeling Individual and Team Behavior through Spatio-Temporal Analysis. In Proceedings of the Annual Symposium on Computer-Human Interaction in Play. Association for Computing Machinery, New York, NY, USA, 601-612. https://doi.org/10.1145/3311350.3347188

[4] Aimdrol. 2020. WoT-Replay-Analyzer. https://github.com/Aimdrol/WoT-ReplayAnalyzer Accessed: August, 2020.

[5] Bang \& Jensen IVS. 2020. Noesis - Interactive CS:GO analytics. https://www. noesis.gg/ Accessed: November, 2020.

[6] Andras Belicza. 2014. Scelight. https://sites.google.com/site/scelight/ Accessed: August, 2020.

[7] Blizzard Entertainment. 2010. StarCraft II. Game [PC]. Nintendo, Irvine, CA, US.

[8] Brian Bowman, Niklas Elmqvist, and T. J. Jankun-Kelly. 2012. Toward Visualization for Games: Theory, Design Space, and Patterns. IEEE Transactions on Visualization and Computer Graphics 18, 11 (2012), 1956-1968. https: //doi.org/10.1109/TVCG.2012.77

[9] Virginia Braun and Victoria Clarke. 2006. Using thematic analysis in psychology. Qualitative Research in Psychology 3, 2 (2006), 77-101. https://doi.org/10.1191/ 1478088706qp063oa

[10] Josh Bycer. 2014. Informed Decisions and their role on Game Design. https://www.gamasutra.com/blogs/JoshBycer/20141217/232510/Informed_ Decisions_and_their_role_on_Game_Design.php Accessed: August, 2020.

[11] Sven Charleer, Kathrin Gerling, Francisco Gutiérrez, Hans Cauwenbergh, Bram Luycx, and Katrien Verbert. 2018. Real-Time Dashboards to Support ESports Spectating. In Proceedings of the 2018 Annual Symposium on Computer-Human Interaction in Play. Association for Computing Machinery, New York, NY, USA, 59--71. https://doi.org/10.1145/3242671.3242680

[12] datdota. 2020. datdota. https://www.datdota.com/ Accessed: November, 2020.

[13] Thomas Debeauvais, Thomas Zimmermann, Nachiappan Nagappan, Kevin Carter, Ryan Cooper, Dan Greenawalt, and Tyson Solberg. 2014. Off with their assists: An empirical study of driving skill in Forza Motorsport 4. In Proceedings of the 9th International Conference on the Foundations of Digital Games. 8.

[14] Anders Drachen and Alessandro Canossa. 2009. Analyzing Spatial User Behavior in Computer Games Using Geographic Information Systems. In Proceedings of the 13th International MindTrek Conference: Everyday Life in the Ubiquitous Era (Tampere, Finland) (MindTrek '09). Association for Computing Machinery, New York, NY, USA, 182-189. https://doi.org/10.1145/1621841.1621875

[15] Anders Drachen and Matthias Schubert. 2013. Spatial game analytics and visualization. In IEEE Conference on Computational Inteligence in Games. IEEE, Washington, DC, USA, 1-8.

[16] Brandon Drenikow and Pejman Mirza-Babaei. 2017. Vixen: Interactive Visualization of Gameplay Experiences. In Proceedings of the 12th International Conference on the Foundations of Digital Games (Hyannis, Massachusetts) (FDG '17). Association for Computing Machinery, New York, NY, USA, 3:1-3:10. https://doi.org/10.1145/3102071.3102089

[17] Meng Du and Xiaoru Yuan. 2020. A survey of competitive sports data visualization and visual analysis. Fournal of Visualization (2020), 1-21. https://doi.org/10.1007/ s12650-020-00687-2

[18] Elo Entertainment. 2020. Dotabuff. https://www.dotabuff.com/ Accessed: November, 2020.

[19] Ensemble Studios. 1997. Age of Empires. Game [PC]. Xbox Game Studios, Redmond, WA, US.

[20] Epic Games. 2017. Fortnite. Game [PC]. Epic Games, Cary, NC, US

[21] William A. Hamilton, Oliver Garretson, and Andruid Kerne. 2014. Streaming on Twitch: Fostering Participatory Communities of Play within Live Mixed Media. In Proceedings of the SIGCHI Conference on Human Factors in Computing Systems. Association for Computing Machinery, New York, NY, USA, 1315--1324. https://doi.org/10.1145/2556288.2557048

[22] Erik Hazzard. 2014. Data Visualization in Games. http://erikhazzard.com/blog/ game-development/data-visualization-in-games Accessed: April, 2020.

[23] Nate Hoobler, Greg Humphreys, and Maneesh Agrawala. 2004. Visualizing competitive behaviors in multi-user virtual environments. In IEEE Visualization. IEEE, Washington, DC, USA, 163-170.

[24] Michael Karlsson. 2016. Information Visualisation in Games: How do you know what you know? Master's thesis. Umea University.

[25] Andrew Karpushin and Clement Durand. 2020. WOTInspector. https: //wotinspector.com/en/ Accessed: August, 2020.

[26] Athanasios Vasileios Kokkinakis, Simon Demediuk, Isabelle Nölle, Oluseyi Olarewaju, Sagarika Patra, Justus Robertson, Peter York, Alan Pedrassoli Pedrassoli Chitayat, Alistair Coates, Daniel Slawson, Peter Hughes, Nicolas Hardie, Ben Kirman, 
Jonathan Hook, Anders Drachen, Marian F Ursu, and Florian Block. 2020. DAX: Data-Driven Audience Experiences in Esports. In ACM International Conference on Interactive Media Experiences. Association for Computing Machinery, New York, NY, USA, 94-105. https://doi.org/10.1145/3391614.3393659

[27] Yen-Ting Kuan, Yu-Shuen Wang, and Jung-Hong Chuang. 2017. Visualizing Real-Time Strategy Games: The Example of StarCraft II. In 2017 IEEE Conference on Visual Analytics Science and Technology (VAST). IEEE, Washington, DC, USA 71-80. https://doi.org/10.1109/VAST.2017.8585594

[28] Yun-En Liu, Erik Andersen, Richard Snider, Seth Cooper, and Zoran Popović. 2011. Feature-Based Projections for Effective Playtrace Analysis. In Proceedings of the 6th International Conference on Foundations of Digital Games. Association for Computing Machinery, New York, NY, USA, 69-76. https://doi.org/10.1145/ 2159365.2159375

[29] Eva Mayr, Nicole Hynek, Saminu Salisu, and Florian Windhager. 2019. Trust in Information Visualization. In EuroVis Workshop on Trustworthy Visualization (TrustVis), Robert Kosara, Kai Lawonn, Lars Linsen, and Noeska Smit (Eds.). The Eurographics Association, 25-29. https://doi.org/10.2312/trvis.20191187

[30] Adam W. Meade and Bartholomew Craig. 2012. Identifying Careless Responses in Survey Data. Psychological Methods 17, 3 (2012), 437-455. https://doi.org/10. 1037/a0028085

[31] Ben Medler. 2013. Visual Game Analytics. In Game Analytics: Maximizing the Value of Player Data, Magy Seif El-Nasr, Anders Drachen, and Alessandro Canossa (Eds.). Springer, London, 403-433. https://doi.org/10.1007/978-1-4471-4769-5_18

[32] Ben Medler and Brian Magerko. 2011. Analytics of Play: Using Information Visualization and Gameplay Practices for Visualizing Video Game Data. Parsons fournal for Information Mapping 3, 1 (2011), 12.

[33] Truong-Huy D Nguyen, Magy Seif El-Nasr, and Alessandro Canossa. 2015. Glyph: Visualization Tool for Understanding Problem Solving Strategies in Puzzle Games. In Proceedings of the 10th International Conference on the Foundations of Digital Games. 9.

[34] OP.GG. 2020. PUBG Stats, Matches, Leaderboards, Guides, Weapons PUBG.OP.GG. https://pubg.op.gg/weapons Accessed: August, 2020.

[35] PUBG Corporation. 2017. PUBG. Game [PC]. PUBG Corporation, Bundang-gu, South Korea.

[36] Riot Games. 2009. League of Legends. Game [PC]. Riot Games, Santa Monica CA, US.

[37] Hans-Jörg Schulz, Thomas Nocke, Magnus Heitzler, and Heidrun Schumann 2013. A Design Space of Visualization Tasks. IEEE Transactions on Visualization and Computer Graphics 19, 12 (2013), 2366-2375.

[38] Magy Seif El-Nasr, André Gagné, Dinara Moura, and Bardia Aghabeigi. 2013 Visual Analytics Tools - A Lens into Player's Temporal Progression and Behavior. In Game Analytics: Maximizing the Value of Player Data, Magy Seif El-Nasr, Anders Drachen, and Alessandro Canossa (Eds.). Springer, London, 435-470. https://doi.org/10.1007/978-1-4471-4769-5_19
[39] Shadow Esports GmbH. 2020. Shadow - Game-changing esports analytics. https: //shadow.gg/ Accessed: November, 2020.

[40] Ben Shneiderman. 1996. The eyes have it: a task by data type taxonomy for information visualizations. In Proceedings 1996 IEEE Symposium on Visual Languages. IEEE, Washington, DC, USA, 336-343. https://doi.org/10.1109/VL.1996.545307

[41] Max Sjöblom and Juho Hamari. 2017. Why do people watch others play video games? An empirical study on the motivations of Twitch users. Computers in Human Behavior 75 (2017), 985-996. https://doi.org/10.1016/j.chb.2016.10.019

[42] Skybox. 2020. Skybox. https://skybox.gg/early-access Accessed: November, 2020.

[43] The OpenDota Project. 2020. OpenDota. https://www.opendota.com/ Accessed: August, 2020.

[44] Valve. 2013. Dota 2. Game [PC]. Valve, Bellevue, WA, US

[45] Günter Wallner. 2018. Automatic generation of battle maps from replay data. Information Visualization 17, 3 (2018), 239-256. https://doi.org/10.1177/ 1473871617713338

[46] Günter Wallner. 2019. A Brief Overview of Data Mining and Analytics in Games. In Data Analytics Applications in Gaming and Entertainment, Günter Wallner (Ed.). CRC Press, Boca Raton, FL, US, 1-14.

[47] Günter Wallner, Alessandro Canossa, and Magy Seif El-Nasr. 2018. Introduction to the special issue on visual game analytics. Information Visualization 17, 3 (2018), 181-182. https://doi.org/10.1177/1473871617722040

[48] Günter Wallner, Nour Halabi, and Pejman Mirza-Babaei. 2019. Aggregated Visualization of Playtesting Data. In Proceedings of the 2019 CHI Conference on Human Factors in Computing Systems. Association for Computing Machinery, New York, NY, USA, Article 363, 12 pages. https://doi.org/10.1145/3290605. 3300593

[49] Günter Wallner and Simone Kriglstein. 2013. Visualization-based analysis of gameplay data - A review of literature. Entertainment Computing 4, 3 (2013), 143-155. https://doi.org/10.1016/j.entcom.2013.02.002

[50] Günter Wallner and Simone Kriglstein. 2016. Visualizations for Retrospective Analysis of Battles in Team-Based Combat Games: A User Study. In Proceedings of the 2016 Annual Symposium on Computer-Human Interaction in Play. Association for Computing Machinery, New York, NY, USA, 22-32. https://doi.org/10.1145/ 29679342968093

[51] Matthew O. Ward, Georges Grinstein, and Daniel Keim. 2010. Interactive Data Visualization: Foundations, Techniques, and Applications (1 ed.). CRC Press, Boca Raton, FL, US.

[52] Wargaming Minsk. 2010. World of Tanks. Game [PC]. Wargaming, Nicosia, Cyprus.

[53] Westwood Studios. 1995. Command \& Conquer. Game [PC]. Virgin Interactive Entertainment, London, UK.

[54] Veronica Zammitto. 2008. Visualization Techniques in Video Games. In Electronic Visualisation and the Arts (EVA 2008). BCS, 267-276. 